\title{
Transición 6 grado a 7año, ¿problema o desafío?
}

\author{
Marianella Castro Pérez. \\ Académica del Instituto de Estudios Interdisciplinarios de la Niñez y la Adolescencia \\ Centro de Investigación y Docencia en Educación, Universidad Nacional \\ Heredia, Costa Rica \\ Mirta Díaz Forbice ${ }^{2}$ \\ Académica de la División de Educación Rural \\ Centro de Investigación y Docencia en Educación, Universidad Nacional \\ Heredia, Costa Rica \\ Hilda Fonseca Solórzano ${ }^{3}$ \\ Académica de la División de Educología \\ Centro de Investigación y Docencia en Educación, Universidad Nacional \\ Heredia, Costa Rica \\ Ana Teresa León Sáenz ${ }^{4}$ \\ Directora del Instituto de Instituto de Estudios Interdisciplinarios de la Niñez y la Adolescencia \\ Centro de Investigación y Docencia en Educación, Universidad Nacional \\ Heredia, Costa Rica \\ Lillian Susana Ruíz Guevara \\ Académica de la División de Educación Básica \\ Centro de Investigación y Docencia en Educación, Universidad Nacional \\ Heredia, Costa Rica \\ Willy Umaña Fernández ${ }^{6}$ \\ Académico de la División de Educación para el Trabajo \\ Centro de Investigación y Docencia en Educación, Universidad Nacional \\ Heredia, Costa Rica
}

\footnotetext{
${ }^{1}$ Licenciada en Educación Preescolar y Máster en Administración Educativa, Académica del INEINA, Universidad Nacional.

Correo electrónico: nella cp@yahoo.com

² Máster en Educación, Académica de la División de Educación Rural, Universidad Nacional.

Correo electrónico: mirtadia@gmail.com

${ }^{3}$ Máster en Educación, Académica de la División de Educología, Universidad Nacional.

Correo electrónico: hildafon@gmail.com

${ }^{4}$ Licenciada en Psicología y Máster en Desarrollo del Niño, Directora del INEINA, Universidad Nacional.

Correo electrónico: analeon@racsa.co.cr

${ }^{5}$ Licenciada en Educación Preescolar, Máster en Administración Educativa y Doctora en Educación, Académica de la División de Educación Básica, Universidad Nacional Costa Rica.

Correo electrónico: susanaruiz@racsa.co.cr

${ }^{6}$ LLicenciado en Planificación Social, Académico de la División de Educación para el Trabajo, Universidad Nacional Costa Rica.

Correo electrónico: educare@una.ac.cr
} 
Recibido 23 de abril de 2009 • Aceptado 20 de mayo de 2009

Resumen. Este artículo despliega los resultados de una experiencia investigativa de dos años, llevada a cabo por un equipo interdisciplinario de las cinco unidades académicas del CIDE. El estudio es parte de un proyecto de investigación más amplio denominado Perfiles, Dinámicas y Desafíos de la Educación Costarricense, el cual es dirigido por el Decanato.

El estudio fue desarrollado durante 2006 y parte de 2007, en colegios identificados como instituciones exitosas con respecto al desempeño académico. La investigación se enfocó en la exploración de los factores que influyen en el desempeño exitoso de los estudiantes cuando ellos transitan de la primaria a la secundaria y a su permanencia durante los años de transición.

La investigación, además de abarcar una revisión literaria importante, requirió trabajo de campo en 10 colegios de diferentes zonas del país. Se realizaron observaciones y se exploraron las creencias de los y las estudiantes de sétimo y octavo año, al igual que las de sus padres, madres y docentes. Además, paralelamente, una importante población de estudiantes de sexto grado, padres, madres y docentes de las escuelas vinculadas con los colegios seleccionados, participaron en el estudio.

Palabras clave. Adolescencia, transición, primaria, secundaria, éxito, factores.

Abstract. The present article displays the results of a two-year research experience carried out by an interdisciplinary research team from the five academic departments (units) at CIDE. The study is part of a larger research project named Profiles, Dynamics and Challenges of Costa Rican education at CIDE, which is directed by the Deanery.

The study was conducted during 2006 and part of 2007 in secondary schools which were identified as successful institutions regarding academic achievements. The research focused on exploring the factors which influence students' academic performance positively as they move from primary to secondary school and as they remain within successful academic indicators during those transition years.

This research, besides involving an important literature review, required field work which took place in ten secondary schools from diverse settings all over the country. Observations were made and the seventh and eighth graders' beliefs, as well as, their parents' and teachers' assumptions were explored. In addition, paralleled to the aspects mentioned, an important population of sixth grade students, their parents, and teachers were studied, since those primary institutions were related to the secondary schools involved in the study.

Key words. Adolescence, transition, elementary school, high school, success, factors.

\section{Introducción}

El Centro de Investigación y Docencia en Educación de la Universidad Nacional desarrolla desde hace varios años, el Programa de Investigación denominado Perfiles, Dinámicas y Desafíos de la Educación Costarricense. La primera investigación realizada en el marco de este Programa, finalizada en el año 2005, llevó a cabo un análisis de la situación educativa en el ámbito nacional. De los resultados encontrados en este primer trabajo, en relación con la deserción ${ }^{7}$ y la repitencia en los diversos niveles, se corroboró la importancia de diseñar y ejecutar un estudio que permitiera comprender, con mayor precisión, cuáles son los factores que facilitan la permanencia de los y las jóvenes en el sistema educativo, particularmente en la transición de la primaria a la secundaria y el impacto en su etapa de desarrollo de acuerdo con el principio establecido por la Convención de los Derechos de la Niñez de la autonomía progresiva.

\footnotetext{
${ }^{7}$ Aunque este documento utilice el término deserción por ser el más familiar, conceptualmente los investigadores de este proyecto visualizan esta situación como expulsión escolar en la que el estudiante no es el único responsable, sino que podrían contribuir los otros actores del sistema educativo.
} 
Las estadísticas reflejan, desde hace muchos años, que los dos niveles con mayor problemática en deserción y repitencia son el primer y el sétimo nivel; momentos en los cuales los y las estudiantes se encuentran en procesos de cambio, adaptación y ajuste, tanto en relación con el sistema educativo como con ellos y ellas en su rol de personas en el ejercicio de su ciudadanía.

Asimismo, se consideró relevante identificar aquellos factores que están asociados al éxito en la permanencia y el avance en el sistema educativo, ya que en otros estudios se habían abordado, principalmente, los factores vinculados a la deserción o al fracaso.

\section{Consideraciones teóricas}

Para efectos de esta investigación, el análisis se plantea dentro del sistema educativo formal costarricense, y abarca, en primaria, el $6^{\circ}$ grado del II Ciclo, y, en secundaria, el $7^{\circ}$ y el $8^{\circ}$ año del III Ciclo de la Educación General Básica; lo que corresponde, según la normativa nacional, al grupo de edad comprendido entre los 12 y los 14 años.

Actualmente, se comparte la creencia de que existe una relación estrecha entre el modelo de sociedad y cultura y la organización política que caracteriza al Estado. Por ello, Dengo (1995), establece que estos ámbitos están en concordancia con los lineamientos educativos y que en tanto ellos varíen, los cambios se verán reflejados en la educación. Igualmente, Bolaños y Molina (2001) señalan que "(...) debe partirse del hecho de que no se puede hablar de educación a espaldas de la sociedad, pues los nexos que los unen son muchos y muy estrechos" (p. 17).

En relación con lo anterior, se realiza un análisis de las estadísticas relevantes con respecto a la tasa de repitencia y deserción de la población adolescente en Costa Rica.

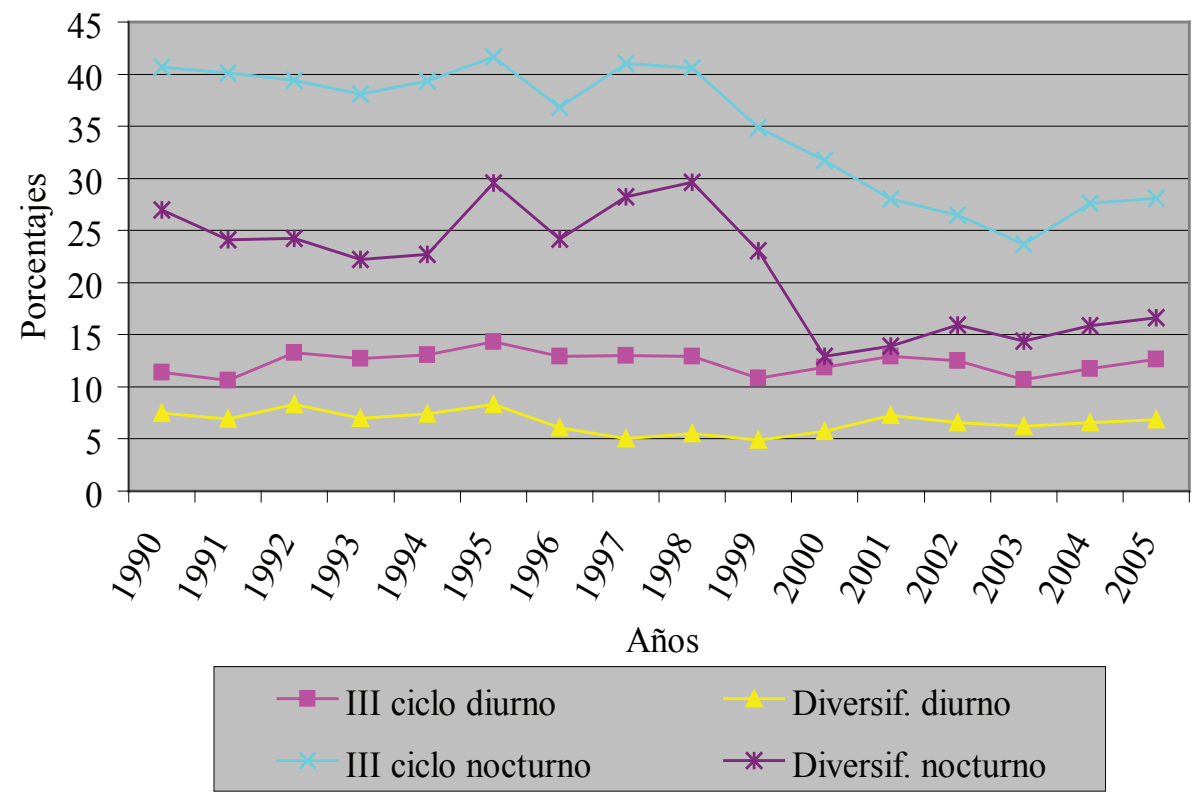

Figura 1. Evolución de la deserción en secundaria en dependencia pública, privada y privada subvencionada, según horario y ciclo. 1990-2005.

Nota. Elaborado por el grupo investigadores, con base en estadísticas del Ministerio de Educación Pública 1990-2005. 
En la secundaria, los mayores niveles de deserción se han venido presentando en III Ciclo y Educación Diversificada con horario nocturno. Los niveles más bajos se presentan en horario diurno; y son más bajos, en el ciclo diversificado.

Por otro lado, los porcentajes de deserción, históricamente, han sido mucho mayores en $7^{\circ}$ y $8^{\circ}$, comparativamente con $6^{\circ}$ grado. Mientras que en $7^{\circ}$ se han mantenido muy constantes y han oscilado entre $15 \%$ y $20 \%$, en el nivel de $6^{\circ}$ grado se han establecido entre $2.8 \%$ y $3.5 \%$, con lo que se han constituido en los niveles más bajos del sistema escolar; es decir, casi no se han modificado en los últimos 15 años.

\section{Repitencia}

La repitencia es otra característica educativa que muestra, también, la eficiencia escolar y son, precisamente, las tasas de repitencia que se presentan en los distintos niveles escolares, los indicadores que permiten su valoración y que determinan la violación del Derecho Fundamental a la Educación.

En la primaria, los mayores niveles de repitencia corresponden al I ciclo y dentro de éste al nivel de $1^{\circ}$ grado, seguido por el $2^{\circ}$ grado, aunque en los últimos años se ha presentado una leve tendencia a disminuir en ambos.

En el II ciclo, se presentan los menores niveles de repitencia de la primaria y en $6^{\circ}$ grado los más bajos y estables, éstos se ubican en alrededor de 1\%; sin embargo, es curioso que al inicio de este ciclo ( $4^{\circ}$ grado) las tasas son más altas que las de $3^{\circ}$ grado, lo que pareciera evidenciar que el cambio de ciclo genera trastornos de rendimiento a los estudiantes.

En la Figura 2, se observa que en la secundaria académica diurna también se presentan las tasas más altas de repitencia al inicio del III ciclo, específicamente, en los niveles de $7^{\circ}$ y $8^{\circ}$. En $7^{\circ}$, las tasas han oscilado entre $14 \%$ y $18 \%$, sobre todo, en los últimos años que ha estado por encima del $16 \%$. En $8^{\circ}$ año, las tasas han oscilado entre $10 \%$ y poco más del $13 \%$, y se ha presentado un ligero incremento al final del período.

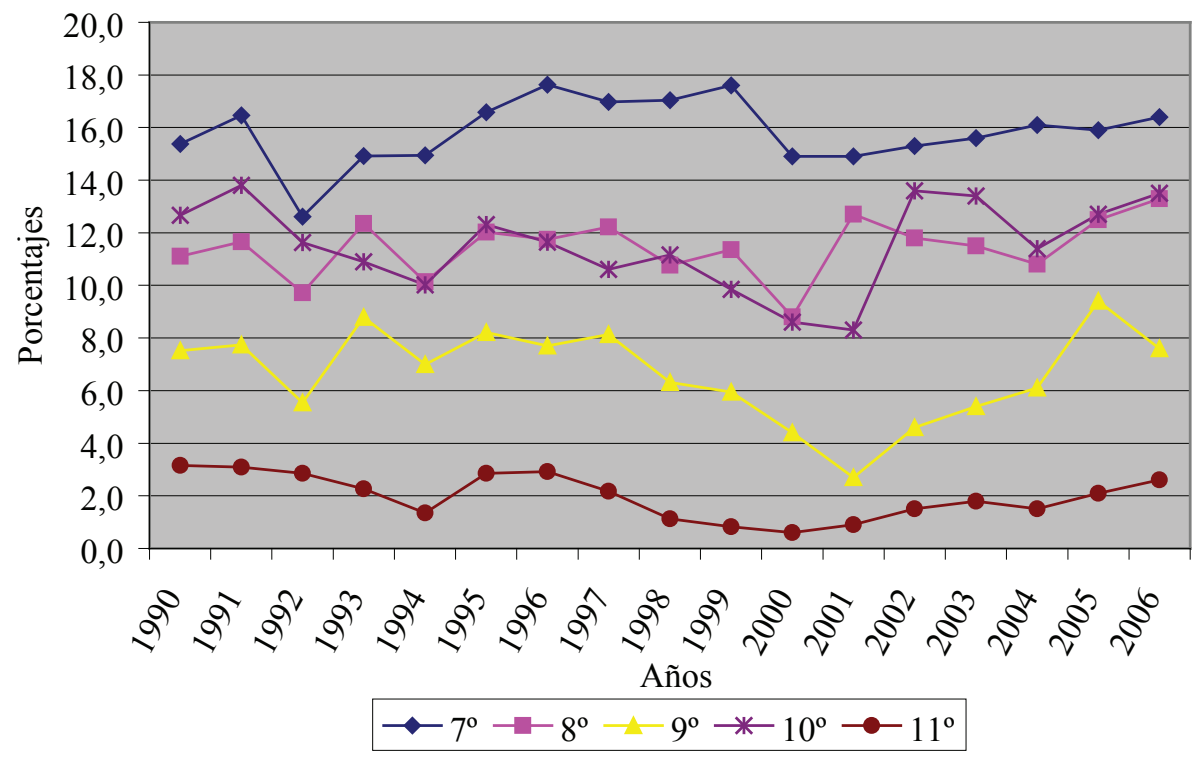

Figura 2. Evolución de la repitencia en secundaria académica diurna en dependencia pública, privada y privada subvencionada, según niveles. Años 1990-2006.

Nota. Elaborado por el grupo investigadores, con base en estadísticas del Ministerio de Educación Pública 1990-2006 
Por último, al compararse las tasas de repitencia de $6^{\circ}$ de primaria y $7^{\circ}$ y $8^{\circ}$ de secundaria, pudo notarse que éstas últimas son mucho mayores. Las tasas de $6^{\circ}$ grado en los últimos años se han ubicado por debajo del $1 \%$, mientras las de $7^{\circ}$ año se han ubicado por encima del $16 \%$ y las de $8^{\circ}$ año por encima del $13 \%$.

\section{Características generales del desarrollo de niños, niñas y adolescentes}

Es necesario plantear que los y las jóvenes que están en la etapa de transición de la escuela al colegio, en realidad, se encuentran en la primera parte de la adolescencia y algunos están aún en la preadolescencia. En este sentido, es importante recordar que los procesos y cambios por los que están pasando los y las adolescentes influyen no solamente en su vida personal, sino, también, en su ajuste, motivación, adaptación y rendimiento dentro del sistema educativo.

Los cambios en la pubertad, etapa que inicia la adolescencia, impactan la vida de los y las jóvenes de diferente manera. La Asociación Nacional de la Escuela Media de los Estados Unidos (1995), ha aclarado que diversos cambios físicos, fisiológicos, emocionales y sociales ocurren en este proceso de desarrollo humano Según Greenberg y Gold (1999), los cambios más dramáticos son los físicos, muchos de ellos originados por los cambios hormonales a los que se enfrentan durante esta etapa.

En el inicio de la adolescencia, existe una relación estrecha entre la apariencia y la socialización, precisamente en el período en que, además, están iniciando la educación colegial. Según Simmons y Blyth (citados por Tokin y Watt, 2003), "para las niñas, la apariencia y la popularidad entre compañeros son elementos importantes para su sistema de valores" (p. 7). Pueden darse situaciones en las cuales prevalece el interés por lograr la aceptación social en lugar de los intereses académicos. También se observa lo contrario, es decir, que la joven que no se considera atractiva o aceptada (y, en muchos casos, los varones también) se refugien en el estudio y se aíslen de la vida social del colegio, por temor a ser rechazados.

En el proceso de transición de la escuela al colegio pueden darse diversas situaciones complejas para los y las adolescentes. Una de estas situaciones se refiere al cambio en la auto-imagen, tanto en el nivel individual como en el grupal. Según Seaton, Egliston, Marsh, Franklin y Craven (2004, p. 1) "algunos estudios han encontrado que el autoconcepto se encuentra en el nivel más alto en el sexto grado, debido a que son los mayores de la escuela primaria y por lo tanto tienen el status más grande"; no obstante, cuando ingresan a la secundaria, pierden dicha condición y pasan a ser los más pequeños, y, muchas veces, sujetos de burlas, bromas y malas experiencias por parte de los más grandes.

De la misma manera, esta etapa coincide con el inicio del desarrollo de la autonomía, especialmente, en relación con la familia, es decir, el paso de la niñez a la vida adulta. Se agrega en el campo social, el cambio en la percepción que se tiene de la familia y, particularmente, de los padres y las madres, quienes empiezan a ser vistos como distantes, diferentes, anticuados y dejan de ser, en muchos casos, referentes de apoyo para los jóvenes en este período de transición. En este sentido, si la relación previa entre niño(a) y adulto(a) fue positiva y de confianza, será más fácil mantener los lazos de comunicación y confianza; si antes de la pubertad, las relaciones eran conflictivas, las condiciones propias de esta etapa solamente agravarán las dificultades. Por lo anterior, también se enfatiza la importancia de darle a los(as) niños(as), especialmente, en los últimos niveles de la primaria, la atención socio-emocional debida, tanto por parte de sus padres y madres como de sus docentes. 
Según Nieda y Macedo (1997, p. 4), "la mayoría de los y las alumnas de 11 a 14 años se encuentra en la transición del pensamiento concreto al formal”. Según los autores de este trabajo, presentan rasgos de ambas etapas, los que se caracterizan de la siguiente manera:

Tabla 1

Características del pensamiento concreto y operaciones formales en adolescentes

\begin{tabular}{|c|c|}
\hline Pensamiento concreto & Pensamiento de operaciones formales \\
\hline $\begin{array}{l}\text { - Operan sobre la realidad concreta, sobre objetos } \\
\text { o representaciones directas de éstos. } \\
\text { - Se ubican en el presente inmediato. } \\
\text { - Son capaces de clasificar y seriar, operando } \\
\text { sobre propiedades observables. } \\
\text { - Reconocen algunas variables que inciden sobre } \\
\text { un problema, pero no logran sistematizarlas. } \\
\text { - No operan sobre enunciados verbales que expre- } \\
\text { sen hipótesis. }\end{array}$ & $\begin{array}{l}\text { - Pueden hacer razonamientos hipotético-deduc- } \\
\text { tivos, deducir conclusiones a partir de hipótesis, } \\
\text { no sólo de observaciones reales. } \\
\text { - Pueden hacer clasificación, seriación y conser- } \\
\text { vación sobre propiedades no observables. } \\
\text { - Pueden combinar ítemes concretos y abstrac- } \\
\text { tos. } \\
\text { - Pueden hacer razonamientos proporcionales, de } \\
\text { correlación y de probabilidad. } \\
\text { - Pueden considerar conjuntos de casos posibles, } \\
\text { tomando uno como real. } \\
\text { - Pueden realizar operaciones a partir de otras } \\
\text { operaciones. }\end{array}$ \\
\hline
\end{tabular}

Nota: Elaborada por los investigadores, con base en una adaptación de Nieda y Macedo (1997).

\section{Concepto y proceso de transición}

En el término transición se conjugan una serie de sucesos, procesos y experiencias, por lo que se dice que resulta impreciso definir este concepto. No obstante, Earwaker (citado por Gimeno Sacristán, 1997) establece una serie de ideas que permiten establecer, de manera más clara, lo que implica este término:

1. Es como un lapso especial en un curso temporal, representa un tramo de tiempo delimitado.

2. La transición denota cambio de ambiente para quienes la experimentan; presupone distancia.

3. Alude a ruptura en la experiencia personal.

4. Resalta momentos críticos en los que se siente perplejidad, inquietud, zozobra.

5. Apela a acontecimientos de los que surgen retos que pueden dejar impronta.

6. Sugiere transformaciones y procesos de adaptación personal que pueden significar consecuencias de futuro en el progreso, en el crecimiento.

7. Señala posibilidades de experimentar traumas.

8. Puede anunciar despegues liberadores. (p. 17)

Las ideas anteriores ejemplifican lo amplio que puede resultar definir transición, especialmente, si se considera que la experiencia de quien la vivencia es también un elemento determinante, sugieren, además, que la transición más que un cambio aislado representa un proceso en el cual el ser humano puede experimentar cambios dramáticos y dolorosos, pero, también, tiene la alternativa de sufrir "despegues liberadores”. Al respecto, Van Gennep (citado por Gimeno Sacristán, 1997) sugiere que como proceso, la transición implica una serie de etapas que las resume en: a) separación del estatus anterior, b) la transición en sí misma y c) la reincorporación o ajuste a la nueva posición 
entre el sujeto y el grupo, es decir, de ahí que el autor afirma que “(...) las transiciones marcan la historia y que ellas mismas tienen una historia: una génesis, un desarrollo y la estabilización en un nuevo estadio" (citado por Gimeno Sacristán, p. 20).

La transición en el ámbito educativo tal y como lo indica San Fabián (2003), puede ser comprendida como un salto curricular con implicaciones culturales "las transiciones son una parte consustancial del sistema escolar, donde avanzar supone un conjunto de escalones o niveles que responden a la propia organización del sistema educativo, que estructura divisiones o parcelas, más o menos arbitrarias, en el conocimiento, en los horarios, en los alumnos, en los profesores" (p. 2). Para este autor, los cambios de nivel en el sistema educativo actúan como ritos de pasaje, es decir, como el tránsito que hace el estudiante dentro de una cultura y que implica "cambio de ambiente, la apertura de nuevas posibilidades, cambio de estatus y un sentido de progresión" (p. 2), por tanto, este rito se caracteriza por ser un momento de crisis.

\section{Factores personales que inciden en la transición de los(as) estudiantes de la primaria a la secundaria: Temores y preocupaciones}

Las creencias, los temores, las expectativas y la motivación en los y las estudiantes se convierten, según se indica en la literatura, en elementos determinantes en el comportamiento y actitud de los y las estudiantes hacia su experiencia de transición, y, por ende, hacia la vida en la secundaria. Kirkpatrick (1997) sugiere que las impresiones y las experiencias iniciales de los estudiantes al ingresar a la secundaria, pueden traducirse en comportamientos difíciles de cambiar "(...) lo que los estudiantes piensan de la secundaria y las cosas que les suceden allí son importantes y afectan la manera en que se comportan académica y socialmente en ese contexto" (p. 1).

Los principales temores que los estudiantes de primaria expresan en relación con su paso a la secundaria, de acuerdo con las investigaciones que aparecen en la literatura (Graham y Hill, 2003, Schumacher, 1998, Anderman y Kimweli, 1997), se refieren a aspectos de índole académico y social, además de que dichos temores se sustentan en el hecho de que el estudiante tiene poca información de la secundaria y, peor aún, los datos que conoce aluden a una experiencia difícil y traumática.

\section{Factores socioafectivos que inciden en la transición}

El componente socioafectivo es, en la actualidad, uno de los temas más polémicos en estudio, especialmente en su relación con la parte educativa. Lo anterior se debe a la complejidad de las relaciones interpersonales entre estudiante-profesor, así como por las demandas de los sistemas educativos, que se centran en el desarrollo de habilidades cognitivas por encima de las sociales. En el caso específico del tema de transición a la secundaria, la literatura establece que la ruptura en las relaciones sociales del o la estudiante, se convierte en una de las consecuencias más importantes en este proceso para los y las estudiantes, de ahí, que sea necesario profundizar en dicha temática.

\section{El rol de la familia}

Tradicionalmente, la familia juega un papel sumamente importante en el proceso de formación de hijos e hijas; sin obviar que, en la actualidad, la constitución de las familias ha variado. 
Las definiciones de familia, por más variadas que sean, descansan hoy en la relación interindividual, y dan la idea de que la familia es, ante todo, un proyecto relacional que no hace referencia, necesariamente, a lazos de sangre. En relación con esto, Schaffer (citado por Luján, 2000) señala que "la naturaleza de las relaciones interpersonales son el factor clave del desarrollo del niño en la familia, más incluso que la propia estructura familiar” (p. 1).

Mizelle (1999), subraya la importancia del involucramiento de los padres y madres con sus hijos e hijas adolescentes. "Cuando los padres están inmersos en la transición de sus hijos ellos tienden a estar involucrados en las experiencias escolares de éstos y cuando los padres están involucrados en las experiencias escolares de sus hijos, los estudiantes tienen un aprovechamiento más alto, un mejor ajuste y son menos propensos a desertar de la escuela" (p. 3).

Además, por ser un derecho de las personas menores de edad se requiere de la responsabilidad estatal para fortalecer las familias que se encuentran debilitadas.

\section{Relaciones entre pares}

En la secundaria, los y las estudiantes, son adolescentes, es decir, no son ni niños, ni tampoco adultos como se suele pensar; por tanto, su mundo y las relaciones que establece, podrían ser complejas, de ahí que la institución escolar se convierta en un refugio para construir un mundo propio al margen de los adultos familiares (Gimeno Sacristán, 1997).

Esta nueva cultura adolescente en la secundaria, hace que los y las jóvenes armen redes de amistad y camaradería, en las cuales los valores de la escuela no son los únicos que pueden encontrar difíciles de seguir, ya que los valores de la familia también se ven enfrentados y, en muchas ocasiones, cuestionados; por tanto, el nuevo grupo de amigos(as) y compañeros(as) se convierte en el signo de seguridad y las relaciones con el sexo opuesto cobran un sentido diferente; además, encuentran mayores espacios para expresarse con amplia libertad, arriesgar iniciativas y relacionarse de igual a igual, sin que existan problemas de mando o superioridad. "En el grupo de iguales el adolescente encuentra lealtad, un clima en el que puede expresar con confianza conflictos y las visiones del mundo de un inseguro yo" (Gimeno Sacristán, 1997, p. 87).

\section{Relaciones con los docentes}

En el proceso de transición de la primaria a la secundaria, los estudiantes experimentan una serie de cambios que incluye entre otras cosas, pasar de relaciones unipersonales con un solo docente, a relaciones más complejas y menos personalizadas con doce o, bien, catorce profesores, quienes, a su vez, interactúan hasta con cuatrocientos estudiantes en una sola semana, razón por la cual, llamar a un estudiante por su nombre o darle seguimiento en su rendimiento académico o personal, pareciera convertirse en una tarea compleja tanto para el docente como para el estudiante, quien debe aprender a independizarse. Esta independencia, desde la perspectiva de Gimeno Sacristán (1997), tiene que ver con el hecho de que los estudiantes, pasan de un sistema maternalista a un ambiente que les exige mayor libertad y control, situaciones que pueden, inclusive, provocar una crisis socioafectiva, en la que debe aplicarse el principio de la autonomía progresiva que establece la Convención de Derechos de la Niñez. 


\section{Rendimiento académico}

En este proceso de transición, las investigaciones confirman que existe una declinación en el rendimiento académico de los estudiantes, lo que los hace experimentar al inicio de este cambio momentos de fracaso escolar, esto se relaciona, directamente, con la cantidad de materias y las demandas de las tareas que les son asignadas. Además, intervienen, según Ruiz (2006), aspectos relacionados con técnicas de estudio inapropiadas, pocas horas regulares destinadas al estudio, mal manejo del tiempo, asuntos de inmadurez personal y psicológica, dificultades propias de las asignaturas y debilidades en el aprendizaje, relacionadas con menos habilidad.

En el caso concreto de Costa Rica, las estadísticas del MEP del año 2005, evidencian que los niveles de aprobación en $6^{\circ}$ grado son muy elevados $(92,7 \%)$ y presenta ligeras diferencias por sexo (92,3\%, en hombres y $93,1 \%$, en mujeres). Sin embargo, cuando los estudiantes ingresan a la secundaria los niveles de aprobación bajan, de manera significativa, en el nivel de $7^{\circ}(72,8 \%$, en general); además, es evidente que se presentan diferencias significativas por género $(69,4 \%$, en hombres y $76,3 \%$, en mujeres). Para el nivel de $8^{\circ}$, los porcentajes de aprobación relacionados con el género, muestran una diferencia de $4.5 \%$ (75,8\%, en hombres y $80,3 \%$, en mujeres). Por tanto, tal y como se establece en la literatura, las diferencias por género son muy evidentes en el rendimiento académico, que presentan los estudiantes.

\section{Factores institucionales que inciden en la transición}

La transición, tal y como lo establece Gimeno Sacristán (1997), refleja una de las discontinuidades que tienen los sistemas educativos, en tanto, no sólo representa, en la mayoría de las ocasiones, un cambio de institución para el alumno, sino que, además, refleja diferencia en términos de la cultura que él vivencia en la escuela y en la secundaria, lo que hace de éstas, dos espacios educativos que no guardan relación entre sí, más que formar parte de un mismo sistema. Por tanto, indica el autor, las soluciones deben ser integrales en términos de mejorar la educación secundaria en general.

Para Antúnez (2005), esta transición, requiere de una serie de consideraciones importantes para que las consecuencias del paso del sexto a sétimo año, sean menos dramáticas para el estudiante. Estas consideraciones se refieren a un trabajo coordinado y de gran compromiso, por parte de los directores de instituciones, docentes, padres y madres de familia y entes ministeriales.

De los planteamientos anteriores, se concluye, entonces, que la transición de un nivel a otro es un proceso y tiene como referente, el sistema educativo en general, de ahí que los factores institucionales van más allá de aquellos que encierran cada escuela o colegio.

\section{Marco Metodológico}

La investigación cuyos resultados se plasman en este escrito, se consideró exploratoria, por constituir uno de los primeros estudios en Costa Rica dirigidos a identificar los factores de éxito en la transición de sexto a sétimo. Sus resultados permitieron lograr un acercamiento o aproximación al tema y sirvieron, además, de punto de partida para otros trabajos de investigación vinculados a esta temática. 
Asimismo, este estudio tuvo un carácter descriptivo, al pretender identificar, a partir de opiniones, criterios y observaciones, cuáles son los elementos o rasgos claves que caracterizan la situación de los colegios considerados más exitosos, en cuanto a permanencia y avance en el sistema educativo. Se utilizaron, en forma complementaria, técnicas cuantitativas y cualitativas; pues, si bien no es representativa de la situación en el ámbito nacional de todos los colegios, se estima que brinda un panorama de cuáles son los factores asociados al éxito en los 10 colegios seleccionados y que están ubicados en diversas zonas de Costa Rica.

\section{Muestra}

Posteriormente al análisis de los datos acerca de los colegios del país, el equipo investigador tomó la decisión de seleccionar los colegios participantes, mediante la combinación de los indicadores baja deserción y alto rendimiento. Además, se tomó en cuenta que estas instituciones estuvieran ubicadas en variadas zonas geográficas, pues se consideró importante tomar en cuenta colegios del área metropolitana, del Valle Central y del resto del país. De esta manera, aunque no se puede hablar de una muestra representativa, sí se procuró una cobertura de todo el ámbito nacional.

La muestra estuvo conformada por: 482 niños y niñas de $6^{\circ}$ grado, 853 adolescentes de sétimo año, 818 adolescentes de octavo año, 16 docentes de sexto grado, 29 docentes de sétimo y la misma cantidad de octavo año. En el caso de los padres y madres de familia (solamente de $7^{\circ}$ y $8^{\circ}$ ), participaron 272 .

\section{Instrumentos}

El equipo investigador preparó instrumentos tipo cuestionario para cada grupo de participantes. Los ítemes obedecieron a una lista de variables preparada sobre la base de la revisión bibliográfica, la experiencia de los investigadores participantes, así como aportes de otros profesores.

Los instrumentos fueron validados con grupos piloto de estudiantes de colegios y escuelas fuera de la lista participante. Esta situación se dio antes de que fueran aplicados a los sujetos de estudio. Los diversos instrumentos incluyeron reactivos, preguntas o mapas conceptuales, de acuerdo con las variables seleccionadas.

\section{Principales hallazgos}

\section{A. Respecto a los características básicas de los diferentes actores}

1. Los 1669 estudiantes de secundaria consultados proceden de 10 colegios académicos públicos con un alto rendimiento y una baja deserción. Prácticamente, la mitad estaba en $7^{\circ}$ año. La mayoría de los estudiantes era del sexo femenino, con edades entre los 12 y 16 años; en especial, los de $7^{\circ}$ año, presentaban sobre edad (más edad de la recomendada para cursar este nivel) y procedían, casi en su totalidad, de escuelas públicas.

2. Los 482 estudiantes de sexto grado que participaron proceden de 10 escuelas consideradas como las que más aportan estudiantes a los colegios seleccionados.

3. De los y las docentes participantes, el $70 \%$ en primaria y el $53 \%$ en secundaria eran de sexo femenino. 
4. Respecto a las familias, los padres y las madres tienden a ubicarse entre los 36 y los 50 años; un 55,9\% de los padres y un 51,2\% de las madres solamente tiene primaria completa o menos, lo cual constituye una desventaja para los y las alumnas de secundaria. Un $68 \%$ de los(as) estudiantes viven con padre y madre; un $26,4 \%$ solamente con la madre. Según los y las docentes, en el nivel de $7^{\circ}$ y $8^{\circ}$, un $40 \%$ es considerado como de condición socio-económica media y un $53 \%$ de baja. Ni una sola familia es considerada extremadamente pobre.

\section{B. Sobre las características de los centros educativos}

1. De los 10 colegios visitados, la impresión general del estado físico reflejó que la mitad de ellos cuenta con condiciones idóneas, mientras que cuatro pueden ser considerados como regulares y uno como deteriorado o malo.

2. Respecto a las áreas recreativas y de juegos, solamente un colegio presentó condiciones óptimas; seis de ellos regulares y tres en condiciones precarias.

3. El mobiliario de las aulas está en buen estado en tres de los centros, en seis está regular y en uno de ellos está en mal estado.

4. La mitad de los centros educativos cuenta con material educativo, $70 \%$ de ellos posee equipo audiovisual suficiente y adecuado.

5. Respecto a las escuelas, la impresión general es que en 8 de las 10 escuelas la infraestructura es apropiada, solamente dos son evaluadas como regulares.

\section{Respecto a los factores asociados al estudio}

1. En cuanto a la actitud de los y las estudiantes hacia el estudio, un promedio de un $21.9 \%$ dice que le gusta mucho estudiar, mientras que un $60 \%$ dice que "más o menos"; por tanto, un porcentaje importante de los y las estudiantes tiene una actitud ambivalente. Las razones más relevantes están vinculadas a materias aburridas, falta de tiempo y pereza.

2. La mayoría de ellos y ellas (73\%) estudia menos de una hora al día; según los y las docentes, la mayoría de los y las estudiantes no estudia lo suficiente.

3. La mayoría de los y las jóvenes manifestó no estudiar mucho y casi la tercera parte, haber repetido algún grado escolar. La mayoría de ellos y ellas fueron estudiantes de buen desempeño en primaria.

4. Se encontró que, aproximadamente, un $67 \%$ de la muestra de estudiantes considera que entiende lo estudiado, un $39 \%$ que se concentra fácilmente y muy pocos necesitan ayuda para estudiar (12\%). Por otra parte, solamente un 38\% de los estudiantes de sétimo y un $31 \%$ de los de octavo demuestra gusto por las clases que imparten los profesores; es decir, solamente una tercera parte de los y las estudiantes disfrutan las lecciones. Las principales razones por las cuales no les gustan las clases es porque son aburridas, por problemas con la metodología o porque no se les explica bien.

5. Poco más de la tercera parte de los y las estudiantes menciona presentar limitaciones para el aprendizaje, entre las más sobresalientes están: el área visual, el área del lenguaje $\mathrm{y}$ el déficit atencional. 
6. Se identifica que los "mejores docentes", tanto para los estudiantes de sétimo como para los de octavo, son los que imparten las llamadas materias básicas, a saber: Ciencias, Español, Estudios Sociales y Matemática.

7. La materia que se selecciona como la más difícil es Matemática, pero, a la vez, es la favorita.

8. Los factores que diferencian a los profesores considerados como los mejores de los peores tienden a centrarse, en sétimo, en las relaciones interpersonales y en la metodología; mientras que en octavo se da a la inversa. Los sétimos valoran la parte humanista de sus docentes, aspecto que pareciera estar relacionado con la necesidad de afectividad, por ser éste el año de transición de un sistema maternal en primaria a uno pluridocente, multimetodológico y multidisciplinar.

\section{En relación con el proceso de transición}

1. La mitad de los y las jóvenes considera que el cambio de primaria a secundaria es brusco, la otra mitad no lo visualiza de esta forma.

2. Las principales dificultades identificadas por los y las estudiantes fueron: los cambios de horario, la cantidad de materias, la cantidad de profesores, el bajo nivel de comprensión de las materias básicas y el temor a incursionar en nuevos grupos sociales. Los factores que más conducen al fracaso, según ellos y ellas, es la falta de interés por el estudio, la indisciplina y el bajo rendimiento. No hacen ninguna referencia a factores económicos, mientras que para los y las docentes lo económico sí es relevante, tanto como la desmotivación o desinterés y los problemas familiares.

3. Para los padres y las madres, los factores más relevantes son el apoyo familiar, el interés de los y las estudiantes por el estudio y la motivación que pueden ofrecer los y las docentes. En síntesis, estudio, apoyo y dedicación constituyen los elementos claramente identificados como críticos para propiciar el éxito y la permanencia en el sistema educativo.

4. Los y las estudiantes de sexto grado consideran que entre los aspectos que les produce mayor preocupación están: el fracaso en los estudios, el nivel de exigencia, la adaptación a las normas sociales y los posibles problemas de violencia. La mayoría de alumnos y alumnas tiene una visión positiva; aunque les gustaría que fuera más alegre y divertido, y que fuera más fácil.

5. En relación con las actividades realizadas para preparar a los estudiantes para el ingreso a secundaria, se concentraron en prácticas para los exámenes, actividades de refuerzo, recuperación y folletos o copias; es decir, la gran mayoría fue académica.

\section{E. Sobre los aspectos socio-emocionales}

1. La mayoría de los y las jóvenes se sienten tranquilos(as), aceptados(as) y alegres. Un porcentaje menor se autodescribe como cumplido(a), disciplinado(a) y seguro(a). Las cuatro características menos reconocidas son: interesado(a), realizado(a), independiente y aplicado(a).

2. Las fortalezas que identifican los y las docentes en los(as) estudiantes de $8^{\circ}$ año se concentran en su colaboración, solidaridad, responsabilidad y deseos de superación. 
3. Respecto a las relaciones interpersonales entre el personal de los centros educativos participantes, parecieran dominar las relaciones interpersonales bastante positivas tanto entre el director, los docentes y el resto del personal administrativo.

4. Las metas y las aspiraciones de los y las jóvenes giran, en gran medida, en llegar a ser profesional; este mismo deseo lo manifiestan los padres y las madres.

5. Los y las docentes subrayan que le dan particular énfasis al componente socio-afectivo; al esfuerzo y a la perseverancia en el estudio y a la obediencia a sus instrucciones.

6. Para los padres y las madres de familia, la educación es importante como medio de superación, esencial para el futuro y también para el desarrollo de sus hijos e hijas; tanto estudiantes como padres y madres aspiran a que completen el Bachillerato. El papel principal de los padres y las madres es el apoyo, la motivación y el seguimiento; cabe mencionar que para cierto porcentaje de ellos este papel es difícil. Para más del $80 \%$ de los y las docentes, el apoyo familiar es crucial para mejorar el rendimiento académico.

7. En síntesis, la población estudiada, considerada exitosa, no documenta porcentajes significativos en cuanto a la existencia de problemas de inasistencia, malas amistades, problemas afectivos o de autoestima, problemas económicos, problemas familiares o falta de apoyo, problemas con docentes o falta de concentración en clase.

8. Los estudiantes considerados como exitosos tienen un autoconcepto muy positivo de sí mismos; lo que más les preocupa es el rechazo de los amigos y no tener buena relación con los profesores.

9. El respeto y el amor son considerados como los valores más importantes en su vida.

10. Cumplir con las tareas encomendadas y ser disciplinados son los atributos que los y las estudiantes más asocian con el éxito escolar.

\section{Sugerencias y propuestas}

\section{Propuestas al Ministerio de Educación Pública}

- Modificar la estructura organizativa y curricular del III Ciclo, dado el poco interés de muchos de los y las jóvenes por el estudio y las repercusiones de esta situación en su rendimiento individual y colectivo. Lo anterior implica pensar los objetivos de la secundaria y llevar a cabo modificaciones significativas en los planes de estudio.

- Abordar los programas de las diferentes materias de tal forma que respondan a las características de estas edades y a los intereses, los derechos y las responsabilidades propias de esta fase de la vida.

- $\quad$ Plantear un perfil del docente de $7^{\circ}$ año y una programación de formación permanente de los educadores; y dar apoyo a los profesores en todo lo relacionado con el proceso de transición a la adolescencia y las características propias de la enseñanza y el aprendizaje en esta etapa.

- $\quad$ Promover en los centros educativos de secundaria, el desarrollo de estrategias que faciliten la transición, tales como actividades de inducción, desarrollo de valores asociados a la solidaridad entre estudiantes, espacios de diálogo con profesores y directores, oportunidades para expresar preocupaciones o temores, entre otras.

- Establecer mecanismos y actividades de preparación, en los centros educativos de primaria, para el ingreso al sétimo nivel. Las sugerencias de los padres y las madres pueden aprovecharse; a continuación se presentan algunas dadas por ellos y ellas: 
- Ofrecer charlas, talleres, convivios, actividades compartidas, conferencias de especialistas, testimonios de personas que han logrado alcanzar éxitos por medio de los estudios.

- Visitar los colegios y que los profesores visiten las escuelas; llevar a los estudiantes de primaria a los colegios y promover actividades conjuntas entre estudiantes de ambos niveles educativos.

- $\quad$ Dar explicaciones sobre el cambio, exponer que ese cambio es bueno, sólo que es una nueva etapa. Ellos y ellas sugieren también procesos de orientación, talleres de formación vocacional, cursos de orientación, atención psicológica, preparación y motivación emocional.

- Dar una buena preparación: con técnicas novedosas, explicar bien las materias, hacer centros, talleres para estimular el pensamiento lógico, mayor calidad de la educación, apoyo en los últimos exámenes, más investigación.

- Realizar actividades recreativas: ferias educativas, bingos, actividades deportivas y culturales compartidas entre estudiantes de sexto grado de escuela y colegiales.

- Motivar a los y las estudiantes por medio de certificados, así como fomentar el entusiasmo de seguir adelante y de ser ellos mismos.

- Llevar a cabo una reorganización administrativa de los sétimo: reduciendo el número de estudiantes por grupo, identificando las materias esenciales y opcionales, permitiendo una etapa de ajuste al III ciclo y no un proceso de adaptación drástico, como lo es para la mitad de los y las estudiantes encuestados.

- Estabilizar la condición de los directores, las directoras y docentes en secundaria, para que puedan desarrollar proyectos de centro de mediano y largo alcance.

- $\quad$ Promover escuela para padres y madres con temática relacionada, tanto para los del nivel de sexto grado en las escuelas aledañas, como a los padres y madres de los jóvenes de sétimo.

- Fortalecer las áreas deportivas y artísticas y otras de interés de los y las adolescentes, con el fin de motivar su permanencia en los centros educativos y desarrollar sus talentos.

- $\quad$ Establecer mecanismos para que aquellos estudiantes que no logren un ajuste o presenten problemas académicos, sean detectados y apoyados, tempranamente, en el año, para que no se retiren de los centros educativos.

\section{Sugerencias para los y las docentes}

- $\quad$ En el caso de los y las docentes de sexto se sugiere: propiciar un ambiente de aula en el cual los y las estudiantes se motiven por aprender, más que por obtener buenas notas. Estos profesionales deben promover estrategias de estudio y análisis que les permitan enfrentar los retos académicos del colegio con bases más sólidas.

- En sétimo: realizar esfuerzos por integrar metodologías y contenidos que propicien un aprendizaje significativo, con el propósito de que los y las jóvenes puedan reconocer la utilidad y la aplicación de los conocimientos y las destrezas que adquieran.

- Contar con espacios para la actualización profesional que implique tanto los aspectos vinculados a la etapa de transición a la adolescencia como el manejo de nuevas 
metodologías, y que en estos procesos se incluya la tecnología. Tener presente el proceso de transición por el que están pasando sus estudiantes, así como las etapas de desarrollo.

- Apoyar a los y las estudiantes con técnicas de estudio, promoción de hábitos y metodologías que les faciliten el manejo y abordaje de tantas materias y contenidos simultáneos.

- Realizar un monitoreo o seguimiento de aquellos estudiantes que estén presentando dificultades o indicadores de fracaso; procurar un equilibrio entre el peso que se da a los componentes académicos y los personales, para que la transición resulte exitosa.

- Cuidar y mejorar las relaciones interpersonales en el aula, estableciendo espacios de diálogo y posibilidad de expresión de sus intereses, motivaciones y preocupaciones, y esforzándose por promover ambientes de confianza y seguridad en las aulas.

- $\quad$ Reconocer la importancia de los aspectos afectivos (autoestima y otros) para el proceso de transición y el impacto que éste pueda tener durante la secundaria.

- Desarrollar la aplicación de los valores en el aula, con el fin de mejorar las relaciones entre estudiantes, así como con el personal docente; reconocer los derechos y las responsabilidades de cada uno de los actores del proceso educativo y promocionarlos entre ellos y ellas.

\section{Sugerencias para los padres y las madres}

- Interesarse en el proceso de transición de sus hijos, por medio de literatura, charlas y mayor contacto con la institución educativa.

- $\quad$ Estar cerca de sus hijos(as) y del Centro Educativo en este proceso de transición, preferiblemente, desde que están en quinto grado, monitoreando y motivando su participación e identificación con esta nueva etapa y con el centro educativo.

- Tener como su rol principal el apoyar a sus hijos e hijas durante el proceso; no obstante, debe buscarse un equilibrio entre la dependencia e independencia y un proceso paulatino de incremento de responsabilidades (autonomía progresiva), a fin de que ellos y ellas asuman su proceso educativo como propio.

- Apoyar los hábitos y las metodologías de estudio de sus hijos, y generar espacios de aprendizaje tanto en lo formal como en lo no formal.

- Promover las buenas relaciones familiares, para que construyan ambientes positivos, afectivos y contenedores; el propiciar la comunicación, el acompañamiento adecuado y oportuno y la confianza son vitales en esta etapa.

- Mostrar interés por los y las adolescentes como personas, estar al tanto de los procesos académicos y sociales de sus hijos e hijas y orientar positiva y constructivamente.

\section{Sugerencias y propuestas para los y las estudiantes}

- Desarrollar estrategias que les permita visualizar la educación no solamente como un medio de superación laboral y económica, sino, también, de realización personal y aprendizaje para y durante toda la vida.

- Comunicarse con el equipo docente y sus familias sobre sus necesidades, para que puedan colaborar en su atención. 
- $\quad$ Promover el desarrollo de hábitos de estudio constante, suficiente y eficaz, y asumir su rol como estudiantes.

- Dialogar con otros estudiantes, así como con adultos sobre el paso al sétimo año; especialmente con respeto al nivel de exigencia y de relaciones interpersonales que ahí se puedan presentar. Fortalecer la capacidad para expresarse y compartir preocupaciones y aspiraciones.

- Solicitar que las instituciones educativas promuevan actividades, visitas y otras alternativas para conocer los colegios a los que vayan a asistir.

- $\quad$ Tener confianza y expresar lo que sienten o sus dudas a sus padres y/o docentes.

\section{Propuestas al CIDE, la UNA y otras universidades}

- $\quad$ Ofrecer propuestas para la transición y para la didáctica en secundaria (como Talón de Aquiles de nuestro sistema educativo).

- Capacitar a los docentes en servicio en el uso de nuevas estrategias y metodologías para el manejo de los contenidos que deben darse en secundaria, que incrementen la motivación y el interés de los y las estudiantes por aprender y permanecer en el sistema.

- Elaborar un perfil del docente de sétimo año congruente con las expectativas del MEP y de la sociedad costarricense, y llevar a cabo una revisión a fondo de los programas que actualmente se ofrecen.

- Formar directores capacitados tanto en la dinámica adolescente, como las características de los procesos de enseñanza y de aprendizaje para este nivel, de transición y la secundaria en general.

- Vincularse con programas de apoyo a las instituciones educativas de secundaria, ofreciendo consultorías y procesos de capacitación que respondan a las necesidades particulares, según el contexto y las características propias de la población adolescente que se atiende.

- Enfatizar durante la formación de docentes de secundaria, los aspectos referidos al manejo de emociones, sentimientos y relaciones interpersonales, como parte fundamental del aprendizaje.

- Capacitar a los profesores de colegio en cuanto a lo que enfrentan los y las jóvenes que vienen de la escuela.

- $\quad$ Ofrecer a los y las estudiantes talleres en distintas áreas: crecimiento personal, aspectos académicos, relaciones interpersonales, entre otras.

- Promover la difusión de los resultados de este estudio y otros vinculados a la problemática en la educación secundaria, y asumir la responsabilidad que corresponde en el mejoramiento de los procesos educativos que se dan en los niveles de secundaria.

\section{Referencias bibliográficas}

Antúnez, S. (2005). El cuido de los procesos de transición de primaria a secundaria: a modo de balance. Aula de Innovación Educativa, 142, 7-11.

Bolaños, G. y Molina, Z. (2001). Introducción al currículo. San José, Costa Rica: Editorial Universidad Estatal a Distancia. 
Dengo, M. E. (1995). Educación costarricense. San José, Costa Rica: Editorial Universidad Estatal a Distancia.

Gimeno Sacristán, J. (1997). La transición a la educación secundaria: discontinuidades en las culturas escolares. Madrid, España: Ediciones Morata.

Greenberg, M. \& Gold, M. (1999). Close and General Friendships among African American, Latino, and Asian American Adolescents from Low-Income Families. Recuperado el 30 de marzo del 2006, de http://spr.sagepub.com/cgi/content/abstract/18/1/29

Kirkpatrick, D. (1997). Making the Change: Students Experiences of the Transition to Primary School. University of Western Sydney. Centre for Learning and Teaching, University of Technology, Sydney. Recuperado el 15 de marzo de 2006, de http//edoz.com.au/educationaustralia/archive/features/make.html

Luján, M. (2000, Octubre). Educación y valores democráticos. Foro Iberoamericano sobre educación en valores. Ministerio de Educación y Cultura, Organización de Estados Iberoamericanos. Recuperado el 20 de junio de http://www.oei.es/valores2/tornaria.htm

Mizelle, N. (1999). Helping Middle School Students Make the Transition into High School. Clearinghouse on Elementary and Early Childhood Education. $\left(\mathrm{N}^{\circ}\right.$ De servicio de reproducción de documentos ED432411).

Nieda, J. \& Macedo, B. (1997). Un currículo científico para estudiantes de 11 a 14 años. Biblioteca Virtual de la Organización de Estados Iberoamericanos (OEI). España. Recuperado el 20 de junio de 2006 de http://www.oei.org.co/oeivirt/curricie/index.html

Ruiz, A. (2006). Universalización de la Educación Secundaria y reforma educativa. San José, Costa Rica: Editorial Universidad de Costa Rica/CONARE.

San Fabián, J. (2003). Coordinación entre primaria y secundaria ;Ojo al escalón! 1 Jornada sobre el tránsit de l'alumnat entre etapes educatives: de l'escola a l'institut Palma de Mallorca. Recuperado el 15 de abril de 2006 de http://weib.caib.es/Documentacio/jornades/jornada transicio/article jlsf.pdf

Seaton, M., Egliston, K., Marsh, H., Franklin, J. \& Craven, R. (2004). Social Comparison: Its Role in the Big-Fish-Little-Pond Effect. SELF Research Centre, University of Western Sydney, Australia. Recuperado el 30 de marzo de 2006, de http://self.uws.edu.au/Conferences/2004 Seaton_Egliston_Marsh_Franklin_Craven.pdf

Tonkin, S. \& Watt, H. (2003). Self-concept over the Transition from Primary to Secondary School: A Case Study on a Program for Girls. Educational Research 13(2) Recuperado el 30 de mayo del 2006, de http://www.iier.org.au/iier13/tonkin.html 